\title{
Design of Ant Colony Optimization Simulation Platform Based On MATLAB GUI
}

\author{
Mengxin $\mathrm{Li}^{1,}$, a) Hongshuang $\mathrm{Hu}^{1, \text { b) }}$, Yong Jiang ${ }^{2, \text { c) }}$ and Guanhua Feng ${ }^{3, \mathrm{~d})}$ \\ ${ }^{1}$ School of Information \& Control Engineering, Shenyang Jianzu University, Shenyang 110168, China. \\ ${ }^{2}$ State Key Laboratory of Robotics, Shenyang Institute of Automation, Chinese Academy of Sciences, Shenyang \\ 110016, China \\ ${ }^{3}$ School of Mechanical Engineering, Shenyang Ligong University, Shenyang 110159, China. \\ a)limengxin@sjzu.edu.cn \\ b)syhgmity@sina.com \\ c)jiangyong@sia.cn \\ d)guanhuafeng_86@163.com
}

\begin{abstract}
In this paper, a novel ant colony optimization (ACO) simulation platform is developed by MATLAB/GUI toolbox to solve traveling salesman problem (TSP). The modular design concept is applied to divide platform into four main modules. The function modules are designed by using MATLAB/GUI toolbox. The development, implementation and test of the designed ACO simulation platform are particularly introduced. The test process and results show that the function of the designed ACO simulation platform can be implemented by importing exiting map and its coordinates or setting coordinates manually; besides, when setting parameters, they can be changed arbitrarily in the range of prompt message. The proposed platform can effectively solve the TSP and find the optimal path.
\end{abstract}

Keywords: design, ant colony optimization, MATLAB/GUI

\section{INTRODUCTION}

Ant colony optimization (ACO) is a promising and satisfying algorithm for excellent performance in solving combinatorial optimization problem [1]. The simulation platform with powerful data processing functions and vivid interface are needed for the realization of ACO. Eyckelhof C J developed a simplified ACO simulation software based on Delphi to serve TSP [2]. The software interface is very simple, but it can only display cities' path state in real-time and its function is too simple. Dr. Haibin Duan designed an ACO simulation platform based on MATLAB, whose interface is improved a lot on the basis of the former [3]. Users can modify parameters as required and monitor the current operational state of program. Users can also switch as required between city state diagram and the optimal path evolution diagram, and even terminal the running program at any time. Ye Chen designed ACO laboratory based on Visual Basic [4]. It not only has all the functions of the previous software and platforms, also increases the average path evolution diagram and other functions, further meeting users' need. Dr Wei Zhou, from the PLA Information Engineering University, developed basic ACO simulation software based on C++ [5]. The dynamic analysis was given in the simulation results. However, these software cannot constraint the setting range of parameters and input method of cities' coordinates is relatively simple. Their results display is mainly based on data without achieving simple and direct effects. 
Based on the above problems and for MATLAB/GUI with the powerful matrix function, good graphic effects and easy modification, the paper presented the development and implement of an ACO simulation platform based on MATLAB/GUI.

\section{FORMULATION OF TSP}

The travelling salesperson problem (TSP) asks the following question: "Given a list of cities and the distances between each pair of cities, what is the shortest possible route that visits each city exactly once and returns to the origin city?"It is an NP-hard problem in combinatorial optimization, important in operations research and theoretical computer science. TSP is a special case of the travelling purchaser problem and the vehicle routing problem.

\section{SYSTEM FRAMEWORK OF SIMULATION PLATFORM}

The system framework always lies at the core of software design. In this paper, the modular design concept is applied to divide modules. The main modules are master control module, input/output module, algorithm module, and simulation module, as shown in Fig. 1.

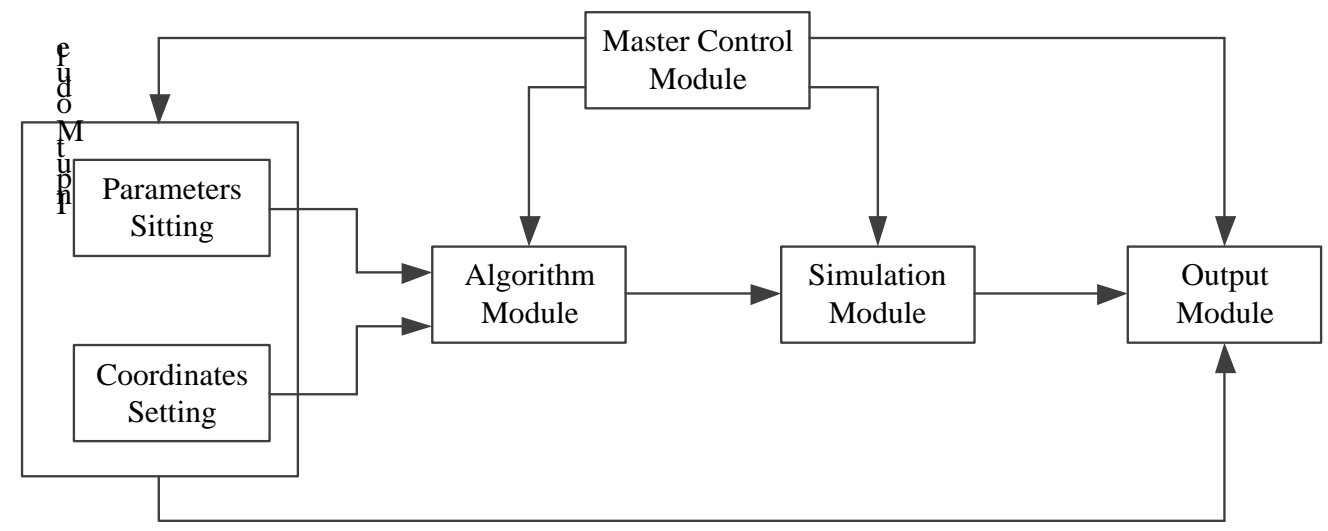

FIGURE 1. Simulation platform system framework.

The input module mainly completes the setting of parameters and the setting of cities' coordinates. After the parameters and coordinates setting are completed, the algorithm module starts to calculate according to the parameters information. The simulation module starts to simulate based on the results from algorithm module. Finally, the output module graphically displays various response curves, numerical results and completes numerical analysis. All of the above modules are coordinated and controlled by the master control module, which can complete the simulation of the entire platform.

\section{DEVELOPMENT AND IMPLEMENTATION OF SIMULATION PLATFORM}

The above function modules of designed simulation platform are designed by using GUI toolbox.

The input module is divided into two parts: parameters setting and city coordinates setting. Parameters setting is used to set the parameters, and it mainly uses the set function to complete assignment. When the parameter is out of the normal range, there will be a prompt message requesting to reset the parameters. The setting of city coordinates can be set as required by using the coordinates input interface or the existing coordinates files. The coordinates input interface is used when users need to set the city coordinates flexibly. And city coordinates and range are not restricted. The algorithm module completes the calculation of the algorithm and controls the start and stop of the algorithm. The simulation module simulates based on the completion of the algorithm calculation. Using axes control and text control, the output module shows the simulation results and data directly. 


\section{TEST OF SIMULATION PLATFORM}

After the development of ACO simulation platform, we test its function. Through the test process, whether the simulation platform can successfully complete the simulation task is verified. With the help of prompt message, the parameters are assigned, as shown in Fig. 2.

Note that information heuristic factor denotes the relative importance of the path and reflects the role of ant's accumulated pheromone during the motion in the process of finding path. The larger the value of the information heuristic factor, the higher probability ants choose the path to move and the stronger the coordination among different ants. Expectation heuristic factor indicates the relative importance of the visibility. The degree in which heuristic information is valued during the motion is reflected. The larger value of the expectation heuristic factor means that the probability of state transition is much closer to the greedy rule.

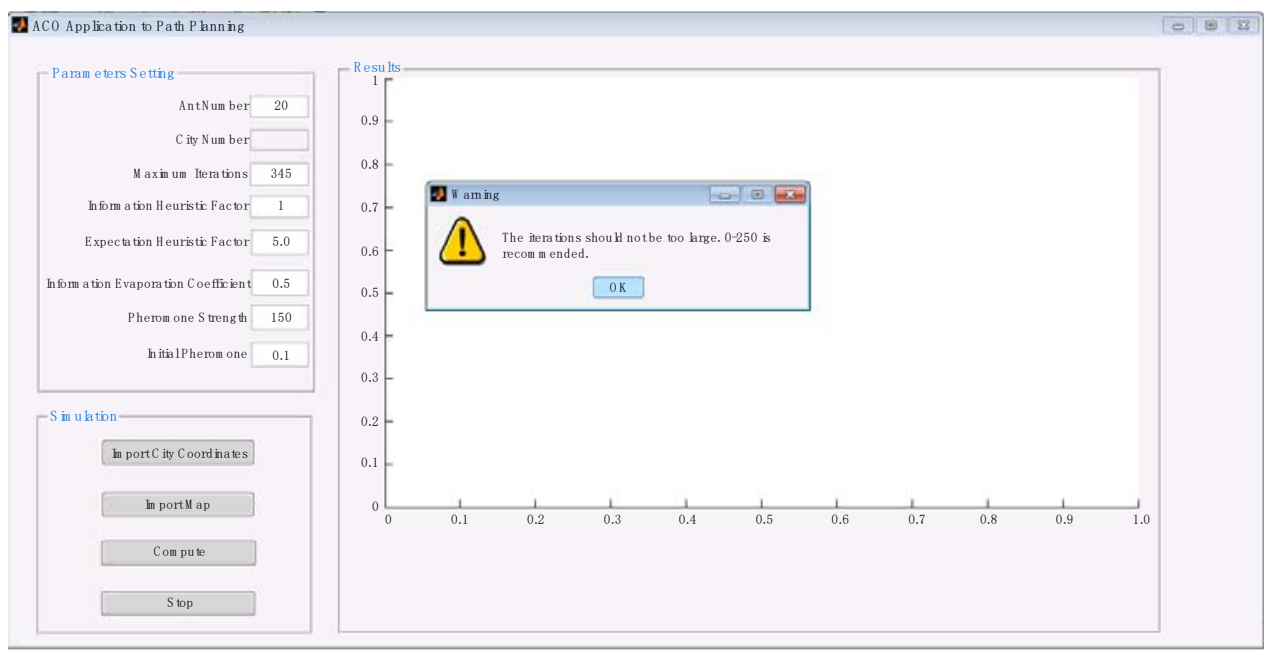

FIGURE 2. Parameters setting.

City coordinates are set by importing exiting map (China's Henan Province), as shown in Fig. 3. Fig. 4 is the calculations by importing map. The test process and results show that the function of the designed simulation platform can be implemented.

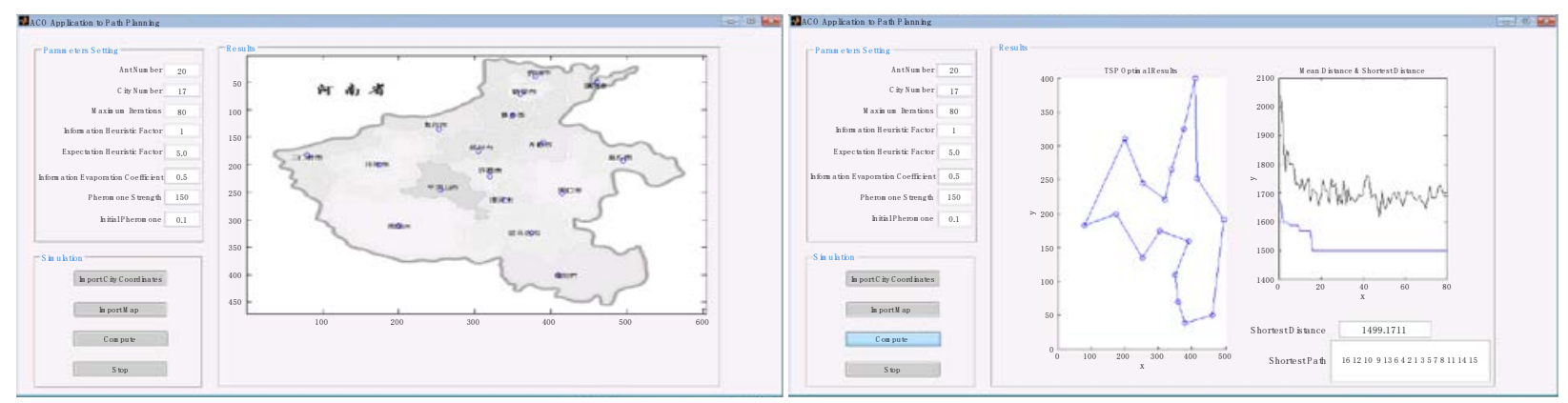

FIGURE 3. Importing map.

FIGURE 4. Calculations.

Based on the parameters setting, city coordinates can be set manually, as shown in Fig. 5. And Fig. 6 is the coordinates distribution. Fig. 7 is the calculations by setting coordinates manually. The test process and results show that the function of the designed simulation platform can be implemented. 


\section{CONCLUSION}

In this paper, combined with the advantages and disadvantages of various programming languages, we develop the ACO simulation platform by MATLAB/GUI toolbox. The graphics display of MATLAB/GUI is convenient. Compared with other programming languages, it has a better effect and is easier for readers to observe. The design, development and implementation of the simulation platform are particularly introduced in this paper. The study concludes as follows,

(1) Modular design concept can allow the simulation platform to choose the way of coordinates input as required.

(2) The function of the designed ACO simulation platform can be implemented

(3) The MATLAB/GUI-toolbox-based ACO simulation platform has a better effect on graphics and results display.

(4) The MATLAB/GUI-toolbox-based ACO simulation platform is very convenient for us to study TSP by changing parameters; besides, the parameters can be changed arbitrarily in the range of prompt message.

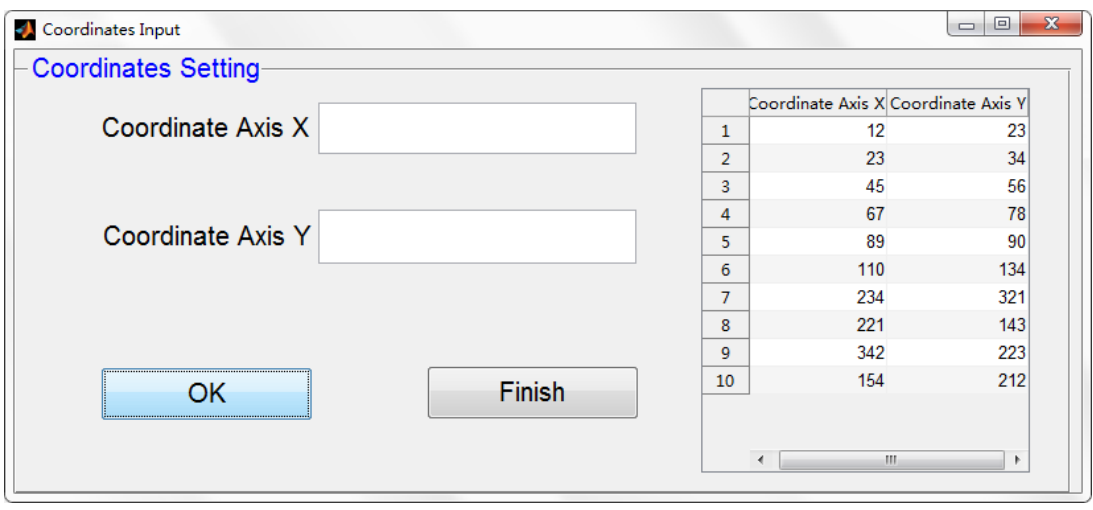

FIGURE 5. Setting coordinates manually.

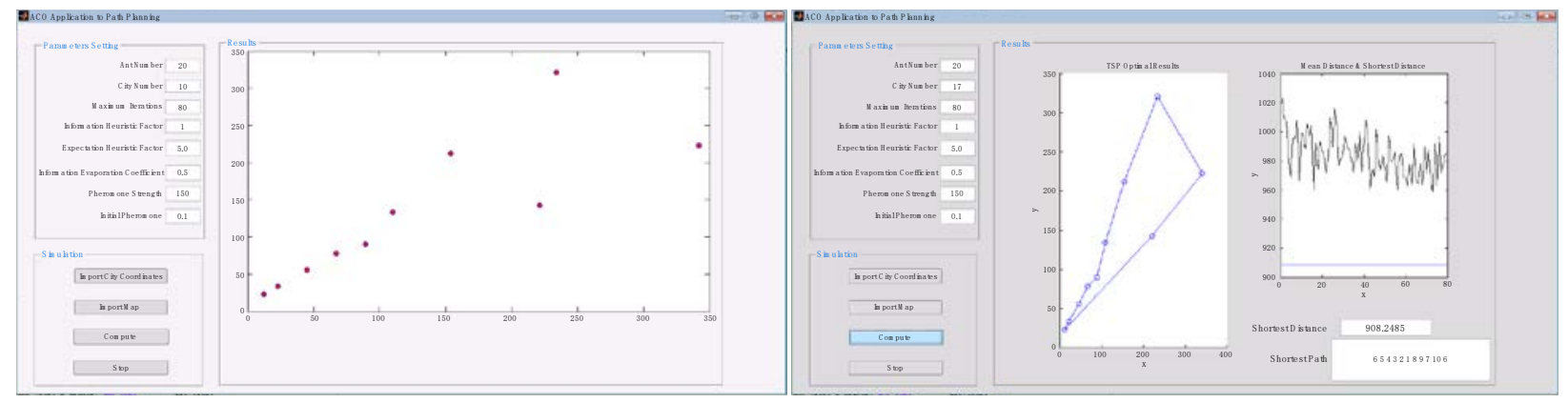

FIGURE 6. Coordinates distribution.

FIGURE 7. Calculations.

\section{ACKNOWLEDGMENTS}

This work was financially supported by the funds from Liaoning Education Department (serial number: LJZ2016012). Corresponding author: Hongshuang Hu.

\section{REFERENCES}

1. Dorigo M, Gambardella L M, Ant colony system: a cooperativel earning approach to the traveling salesman problem. J. EI. Commun. 1 (1997) 53-66.

2. H.B. Duan, Principle and Application of Ant Colony Algorithm, Science Press, Beijing, 2005. 
3. H.B. Duan, D.B. Wang, X.F. Yu, Ant Colony Algorithm: Survey and Prospect. J. Commun. 19 (2004) 13211326.

4. X.Y. Xia, Y.R. Zhou, Adcances in Theoretical Research of Ant Colony Optimization, J. Commun. 11 (2016) $1-13$.

5. L. Zhang, X.Y. Liu, Z.Q. Li, Clustering Optimization Based on Ant Colony Algorithm, J. Commun. 36 (2010) 190-192. 